\title{
A Funny Thing Happened on the Way to the Hospital
}

Jeffrey R. Alberts

Indiana University, NFI Science Committee, Associate Editor for Science

DOI: $10.14434 /$ do.v13i1.29094

Target article: Montirosso, R. and Provenzi, L. (2015) Implications of Epigenetics and Stress Regulation on Research and Developmental Care of Preterm Infants. JOGNN, 44, 174-182. DOI: 10.11 11/1552-6909.12559

$\mathrm{B}$ odies of knowledge, like 'flesh-and-bone' bodies, work best when they are in balance. Today, many bodies of biomedical knowledge are out of balance. For example, the body of knowledge comprising mechanisms of pain and pleasure is large and detailed on the side of pain mechanisms, but knowledge about how pleasure works is sparse and incomplete. Similarly, we study depression more than happiness. In all, we know much more about processes of sickness than of health. There is a pervasive lack of balance!

Biomedical knowledge has distinctly practical, "applied" importance. This kind of knowledge is created, tested, translated, vetted and then sent off to work in hospitals, clinics and other healthcare settings. On its way to the hospital, imbalances appear. Why are bodies of knowledge so lopsided?

Behavioral epigenetics, a field that is nicely introduced by the review article, "Implications of Epigenetics and Stress Regulation on Research and Developmental Care of Preterm Infants" (Montriosso \& Provenzi, 2015) provides some insight into the way we define our areas of study and how this shapes our questions and hence the subsequent shape of our bodies of knowledge. It's a fine paper, worth reading and understanding.

Behavioral epigenetics recently burst onto the science scene as a major disrupter. It's great to shake things up, and epigenetics does it by challenging the "conventional wisdom" about the relations between genotype and behavior. In the process, epigenetics is re-writing basic rules of inheritance, by making obsolete phrases such as "genetic programs", or "there are genes for ...", or "it's in their DNA to ...". The way that epigenetics forces a change in our vocabulary and thinking is that it reveals mechanisms whereby gene expression changes, without the actual genome changing! This is accomplished, as the authors explain, via molecular mechanisms that act "above" the genome (hence epi-genetic). Several such mechanisms are now known.

One of the most commonly studied epigenetic mechanisms is methylation, a process that "silences" gene expression at specific sites, usually within regulatory regions of gene where a methyl group essentially grabs onto a site and prevents DNA $\rightarrow$ RNA transcription. In contrast, histone modification is a molecular mechanism that promotes gene expression. Histone modification involves an acetyl group acting to unwrap a histone (protein) "tail" in a way that exposes DNA to transcription factors and thus facilitates gene expression. Again, Montriosso and Provenzi do an admirable job describing and illustrating some of these molecular events that comprise the epigenetic "marks" that can be assayed after various laboratory manipulations of an animal or life events in humans.

Noted in the target article is a well-known example of epigenetic change. The research originated in the McGill University laboratory of Michael Meaney, where he and a group of associates analyzed different styles of maternal behavior in rats. Some mother rats (also called "dams") spent lots of time licking and cuddling their infants whereas others reared healthy babies but provided much less licking and cuddling. Although equivalent in growth and viability, the offspring of the attentive dams grew up to modulate their stress reactions and display resilient recovery from stress far faster than the offspring of the inattentive dams. When they became mothers, the female offspring of the two types of rat dams (attentive and inattentive) displayed similar styles of mothering. Mothering style and its effects on offspring had been "inherited". Shocking to some, however, were the results of cross-fostering experiments: When attentive moms reared the babies of inattentive moms, those offspring showed the calmer, stress resilient demeanor whereas offspring of attentive dams reared by inattentive mothers developed into stressedout, poorly regulated rats. Moreover, when these females became mothers, their mothering style was that of the foster dam, not the biological mother! Yes, mothering style was "inherited" across the generations, but not via the mother's genes (see Champagne \& Curley, 2009; Meaney, 2001).

It was possible to examine neurons in various brain regions of the offspring reared by dams with the distinctly different mothering styles. One dramatic difference was the density of glucocorticoid receptors (GRs) on the neurons in the hippocampus, a brain structure associated with emotion and learning. The studies surrounding this finding indicate that mothering styles by the rats primed the stress responses (described in the target article in terms of the hypothalamic-pituitary-adrenal or HPA activity) of the infants. The baby's stress responses to the maternal environment, whether from the biological or the foster mom, altered the GR density on the hippocampal neurons and, as a result, altered responses to their own stress hormones. The researchers have found increased methylation at the sites responsible for GR production, pointing the way to the epigenetic shaping of the offspring's brain.

There are now studies in humans showing results compatible with this rat research. For instance, Lester et. al. (2018) studied healthy, 5-month-old, term infants and their mothers, who either did or did not breastfeed exclusively to the time 
of the experiment. They hypothesized that the breastfeeding relationship would serve as a maternal behavior difference to parallel the two groups dams used in the rat research. Lester's group staged a slightly challenging mother-infant interaction (a "still face" test) and measured the babies' stress reactivity via salivary cortisol. They also measured DNA methylation in the GR gene region of the babies' DNA from cheek swabs. Breastfeeding by the human babies was associated with lower DNA methylation and decreased cortisol reactivity. The results provided a striking replica of the rodent studies!

There's another stunning aspect of epigenesis: Epigenetic changes can be inherited! Epigenetic effects are shown to be associated with specific physiological or behavioral traits, typically caused by different types of experience within a lifetime, and then the same epigenetic marks are inherited from the adult via the DNA in the gametes, so that the epigenetic changes are passed to their next generation. But the DNA content of the cells has not been modified. This is transgenerational inheritance with no genetic change! It's a new view of inheritance.

It is a privilege to write a commentary about a new area of knowledge creation. Hopefully, I've conveyed a sense of excitement about epigenetics and inspired you to read the Montriosso and Provenzi paper, and maybe more on the topic. Nevertheless, I've also lamented an imbalance in biomedical knowledge. I argued that there has developed a culture that emphasizes studying sickness more than understanding health. This imbalance is perpetuating because each preliminary discovery leads to related questions which favors more and deeper questions about sickness and not about health. The field of behavioral epigenetics is nascent and just taking shape. Already, the weight of the evidence-based knowledge is accumulating on the side of toxic stress, effects of poor parenting, bad diet, and other negative factors and forces. Framing the issues this way naturally leads us to seek ways to prevent or undo harm. Yes, there is merit to this, but I believe there is more to promoting health than blocking or remediating damage. There are paths to travel, on which we can acquire knowledge that can deepen our ability to guide, facilitate and maintain healthy development. Many of these paths course through NICUs.

Imagine bodies of knowledge growing and developing on their way to being implemented in the hospital. What if we not only understood how surges of adrenal hormones in combination with painful stimuli silence genes that are part of diminished stress reactions, but we also mapped the epigenetic marks of resilience to adversity, or we could reveal the ways in which NIDCAP facilitates histone modifications associated with a premie's ability to self-regulate?

What if we discovered that processes labelled as remedial or protective are identical to formative accretions in normal development? We might discover that a funny thing happened on the way to the hospital: We shifted emphasis and we lost sight of healthcare and instead saw mainly sickcare. Perhaps we can use behavioral epigenetics as a field with new pathways, ones that balance studies of health and disease. Indeed, there are instances and insights into the basis of healthful development. These appear in some studies of mother's milk and the development of immune competence, we see examples across the landscape of microbiome research, and in some studies of oxytocin and related neuropeptides in development. The promotion of "nurture science" (Bergman et al., 2019) is encouraging. Indeed, about a decade ago, Professor Heidelise Als wrote an instructive and insightful article in the Developmental Observer (Als, 2011), in which she foresaw the important promise and implications of epigenetics on NIDCAP. I recommend reading Montriosso and Provenzi (2015) along with Als (2011). To continue your education, go on to read the remarkable and beautifully crafted book by David S. Moore (2015) on behavioral epigenetics.

References

1. Als, H. (2011) Lamark, Darwin and the Science of NIDCAP: Epigenetics in the NICU. Developmental Observer, 4 (2), 1-4.

2. Bergman, N.J., Ludwig, R.J., Westrup, B. Welch, M.G. (2019) Nurturescience versus neuroscience: A case for rethinking perinatal mother-infant behaviors and relationship. Birth Defects Research. 1-18. https://doi.org/10.1002/bdr2.1529

3. Champagne, F. A., \& Curley, J. P. (2009). Epigenetic mechanisms mediating the long-term effects of maternal care on development. Neuroscience and Biobehavioral Reviews, 33(4), 593-600. doi: 10.1016/j.neubiorev.2007.10.009

4. Lester, B.M., Conrad, E., LaGasse, L.L., Tronick, E.Z., Padbury, J.R., Marsit, C.J. (2018) Epigenetic programming by maternal behavior in the human infant. Pediatrics, 142(4), e20171890

5. Meaney, M.J. (2001) Maternal care, gene expression, and the transmission of individual differences in stress reactivity across generations. Annual Review of Neuroscience, 24, 1161-1192.

6. Moore, D.S. (2015) The Developing Genome: An Introduction to Behavioral Epigenetics. Oxford University Press. https://www.amazon.com/Developing-Genome-IntroductionBehavioral-Epigenetics/

\section{NIDCAP Care in the Moment}

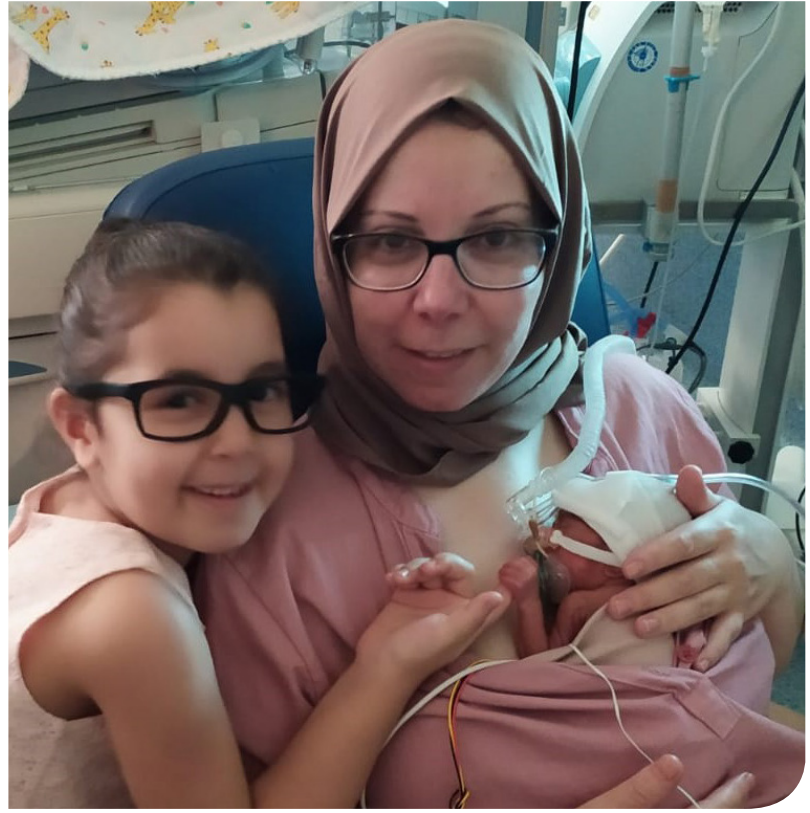

Sibling care 